\title{
INJUSTICIAS AMBIENTALES EN COLOMBIA: ESTADÍSTICAS Y ANÁLISIS PARA 95 CASOS
}

\author{
Environmental Injustice in Colombia: \\ Statistics and Analysis for 95 cases
}

\author{
MARIO ALEJANDRO PÉREZ-RINCÓN ${ }^{1}$ \\ 1Profesor Universidad del Valle - Instituto CINARA, Cali, Colombia. \\ Calle 13 No. 100-00, Ciudad Universitaria Meléndez, Edificio 342.
}

E-mail: mario.perez@correounivalle.edu.co

Recibido: 7 de Octubre de 2014

Aceptado: 15 de Noviembre de 2014

\section{Resumen}

Las políticas de liberalización de mercados a inicios de los noventa contribuyeron a conservar el rol histórico de Colombia en la división internacional del trabajo como exportadora de materias primas y recursos energéticos, expandiendo las fronteras económicas de esas actividades. Esta expansión se encontró con formas de producción campesina y sociedades híbridas que dependen en buena medida de los servicios ambientales provistos por la naturaleza, con lo cual se intensificaron en forma significativa los conflictos e injusticias socio-ambientales. En este contexto, el propósito de este artículo es doble: i) realizar un inventario y mapeo de los principales conflictos ambientales en Colombia, habiéndose documentado inicialmente 95 casos; ii) realizar un análisis descriptivo de los conflictos ambientales a partir de las principales variables utilizadas en el estudio. Los resultados de la investigación permiten concluir que el sector extractivo explica buena parte de los conflictos ambientales; que existe una clara relación entre la cantidad e intensidad de los conflictos y el modelo de desarrollo extractivo de los últimos gobiernos; que los principales grupos afectados son las comunidades pobres; que existe gran dualidad para resolver los conflictos: por un lado la violencia persistente y por otro, el uso de los mecanismos institucionales y legales. Esto muestra la bipolaridad de la sociedad colombiana que se enmarca en los extremos entre negociación, juridicidad y violencia.

Palabras claves: Conflictos socio-ambientales, Justicia ambiental, Reprimarización, Metabolismo social.

\section{Abstract}

The policies of market liberalization in the early nineties contributed to preserve the historical role of Colombia in the international division of labor as an exporter of commodities and energy resources, expanding economic borders of those activities. This expansion encountered forms of peasant production and hybrid societies that depend largely on the environment services provided by nature, which significantly intensified conflicts and socioenvironmental injustices. In this context, the purpose of this article is twofold: i) to inventory and mapping of the main environmental conflicts in Colombia, having initially documented 95 cases; ii) a descriptive analysis of environmental conflicts from the main variables used in the study. The research results support the conclusion that the extractive sector explains much of environmental conflicts; there is a clear relationship between the amount and intensity of conflicts an extractive development model of recent governments; the main groups affected are poor communities; great duality exists to resolve conflicts: one persistent violence and secondly, the use of institutional and legal mechanisms. This shows the bipolarity of Colombian society which is part of the extremes of negotiation, legality and violence.

Keywords: Socio-environmental conflicts, Environmental justice, Reprimarization, Social metabolims. 


\section{INTRODUCCIÓN}

Las políticas de liberalización de mercados en América Latina y el Caribe (ALC) a inicios de los noventa, que surgieron como resultado del llamado Consenso de Washington y fueron promovidas por los organismos financieros internacionales, contribuyeron a un proceso de reespecialización productiva hacía el sector primario y a la pérdida de dinámica del sector industrial en la economía de la región. La reprimarización de las economías latinoamericanas, mantiene el papel de liderazgo en la generación de divisas o de recursos para el Estado del sector extractivo, conservando la región su rol histórico en la división internacional del trabajo como exportadora de materias primas y de recursos energéticos para el desarrollo del proceso metabólico del Sistema Económico Mundial (Wallerstein 1974, Hornborg 1998).

Dado el carácter intensivo y extensivo de estos modelos extractivos en términos del uso de la tierra, el agua y la naturaleza, se generan grandes niveles de acumulación de los recursos naturales, y con ello grandes impactos y conflictos ambientales entre los nuevos actores empresariales que expanden sus actividades bajo patrones agresivos en términos tecnológicos y de vida, y las comunidades que tienen otra forma de relacionarse con la naturaleza y entre sí mismos. La expansión de estas actividades se encuentra frecuentemente con formas de producción campesina, con agricultura familiar, mezcla de cultivos para el autoconsumo y el mercado local, con sociedades híbridas que dependen en buena medida de los servicios ambientales provistos por la naturaleza y con poco respaldo institucional.

Esta realidad, hace a las comunidades más vulnerables, con lo cual la expansión de tales actividades potencia los impactos socio-ambientales sobre las mismas, incrementando la exclusión hacia el uso y disfrute de los recursos naturales de los cuales subsisten, afectando sus modos y medios de vida, sus redes sociales, sus estructuras culturales y sus derechos consuetudinarios sobre los bienes comunes de los cuales dependen altamente, generándose un despojo del agua, la tierra, los territorios comunales, la biodiversidad, etc. Pero además, esta expansión económica se encuentra con territorios ricos en biodiversidad, por lo cual muchos grupos ambientalistas e instituciones también protestan en su defensa.

Partiendo de este contexto, el propósito de este artículo es realizar un inventario inicial de los principales conflictos socio-ambientales en Colombia, caracterizarlos y establecer relaciones analíticas que ayuden a entender sus orígenes y sus efectos.
Para alcanzar este objetivo el artículo está organizado como sigue: en una primera parte se presenta la dinámica económica del país para fundamentar el proceso de especialización productiva hacia el sector primario y sus correspondientes implicaciones en términos socioambientales; posteriormente se muestra el proceso y la metodología desarrollada para seleccionar, acopiar y recoger la información de los diferentes conflictos analizados; en el tercer punto, se presentan los resultados de la investigación en términos del inventario de los conflictos, su análisis descriptivo y una taxonomía clasificatoria de los mismos. Finalmente se entregan las principales conclusiones y la bibliografía consultada.

\section{LA REALIDAD REPRIMARIZADORA DE COLOMBIA Y SUS IMPLICACIONES AMBIENTALES}

Algunos datos estadísticos para Colombia muestran la realidad del proceso que algunos autores han denominado reprimarización de las economías (Nadal 2011), y otros lo conocen como neo-extractivismo (Gudynas 2010, 2011, Svampa, 2013). Así, del crecimiento total de las exportaciones entre 1990 y 2013, que alcanzó US\$ 97583 millones, las ventas al exterior de origen primario aportaron casi el $68 \%$ de este monto, cifra que asciende al $84 \%$ del total de bienes exportados cuando se incluyen las exportaciones manufactureras de origen primario (www.eclac.cl/comercio/SIGCI/). De esta manera, la participación de las exportaciones totales asociadas a materias primas en Colombia pasó de 77,8 a 84\% entre 1990 y 2013 (Figura 1).

Pero además, se han producido importantes cambios estructurales al interior del sector primario exportador. Así, mientras a finales de la década de los setenta, el café representaba el primer renglón de exportación con más del $60 \%$, perdió participación en las siguientes décadas hasta caer a un reducido 3\% en 2012. Al tiempo, el sector mineroenergético ganaba en importancia exportadora. En el mismo periodo, el petróleo y sus derivados pasaron de 8 a más del $50 \%$ del total de ventas al exterior, mientras el carbón y el ferroníquel alcanzaron para 2012 el 12 y $2 \%$ respectivamente. En síntesis, en este año el $64 \%$ de los productos exportados provienen del sector minero-energético, evidenciando el proceso de especialización hacia este sector extractivo.

Esta dinámica reprimarizadora se refleja igualmente en la estructura sectorial del PIB. Así, aunque en el largo plazo se produce una caída del PIB extractivo (del 28 al 16,8\% entre 1970 y 2013), en la última década se genera una recuperación: de $12 \%$ en 2000 a casi $17 \%$ en 2013 (Figura 2). Además, se produce una reestructuración al interior del PIB primario: 
mientras que el sector agropecuario pasó del 23\% en 1975 al $6 \%$ en 2012, el sector minero-energético incrementó su participación en el PIB del $2 \%$ al $11 \%$ en el mismo periodo (DANE 2013).

\section{0}

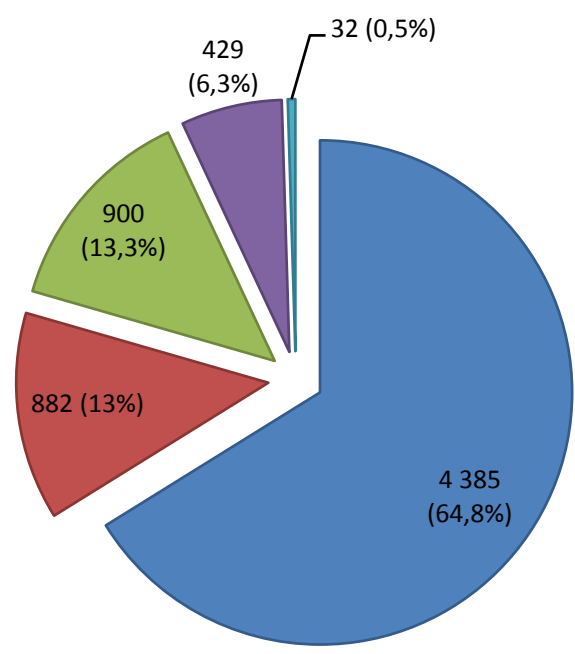

2013

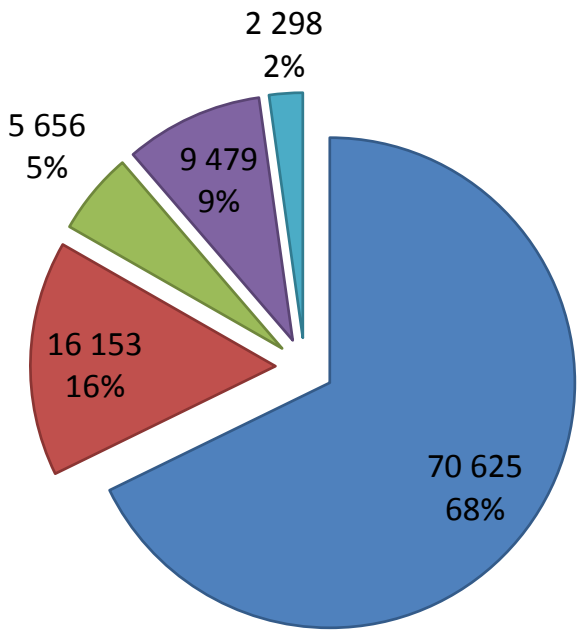

Figura 1. Exportaciones de bienes por categoría de productos, Colombia. Millones de US\$ y \% (1990 y 2013). Fuente: CEPAL: www.eclac.cl/comercio/SIGCI/.

Es importante decir que estos resultados no han dependido solo de fuerzas económicas sino de decisiones políticas; para llegar a la situación neo-extractivista actual se han tomado una serie de decisiones claves en los últimos años. Para América Latina, la década de 1990 implicó grandes reformas en los sistemas económicos y políticos que se reflejaron en diversos ámbitos. En cuanto a la minería, la mayoría de países hicieron reformas a los Códigos y Leyes Mineros, adecuándolos a las nuevas condiciones del mercado, favoreciendo la liberalización y la inversión extranjera (Fuentes 2012).

En el caso de Colombia, las reformas se iniciaron en 1988 con el primer código de minas (Decreto 2655), que se sustentó en cuatro elementos principales: i) Estableció que los recursos mineros sean Patrimonio de la Nación; ii) Constituyó las primeras empresas mineras de orden estatal; iii) Dio vía libre a los títulos mineros; y, iv) Calificó a la industria minera como de interés social o de utilidad pública (Fierro 2012).

De tal manera, entrando el presente siglo, las condiciones para el desarrollo del sector extractivista estaban servidas. Tenían un marco legal que facilitaba el proceso. Faltaba un impulso asociado a las políticas y este llegó con el primer gobierno de Uribe Vélez (2000-2004), las cuales han tenido continuidad hasta hoy. Estas políticas se sintetizan en los incentivos a la inversión nacional y extranjera a partir de exenciones y descuentos tributarios, flexibilización laboral y ambiental, expansión de la oferta de concesiones territoriales o títulos de explotación para minería, petróleo y cultivos agroexportadores, acuerdos de protección a las inversiones y firmas de tratados de libre comercio. Los resultados de ello son evidentes: la inversión extranjera pasó de US\$ 1446 millones en 1994 a US\$ 16772 millones en 2013, jalonado principalmente por la dinámica inversionista hacía los sectores extractivos (minería, petróleo, agricultura, energía, gas y agua), los cuales pasaron de representar el $14 \%$ al $51 \%$ del total de la inversión foránea en el mismo periodo (Banco de la República: www.banrep.gov.co).

\section{METÓDOS Y HERRAMIENTAS PARA LA IDENTIFICACIÓN Y RECOPILACIÓN DE LOS CASOS DE CONFLICTOS AMBIENTALES}

La recopilación de los casos se hizo a partir de un trabajo con estudiantes, ONG's, centros académicos e investigadores, observatorios de conflictos, revisión de noticias de prensa y de páginas web, consultas con los afectados y visitas de campo, mediante los cuales se identificaron los conflictos ambientales. A partir de esta primera identificación, se recogió información para cada conflicto a través del formulario que el proyecto EJOLT (Environmental Justice Organization, Liabilities and Trade) diseñó para ello.

Una vez revisada la pertinencia y calidad de la información de cada caso, a través de actividades de planeación participativa con el Grupo de Investigadores, se identificaron, 
clasificaron, jerarquizaron y analizaron cada conflicto ambiental del país. La jerarquización de los conflictos se realizó a través de un taller de Decisión de Grupo usando metodologías como el brainstromirng (Osborn 1953) y nominal grouptechnique (Delbecq \& Van der Ven 1971), donde cada experto valoró la incidencia de cada conflicto a partir de determinados rangos de valoración. Ello permitió generar una matriz grupal que facilitó la jerarquización de los conflictos, seleccionando en forma definitiva 95 conflictos.

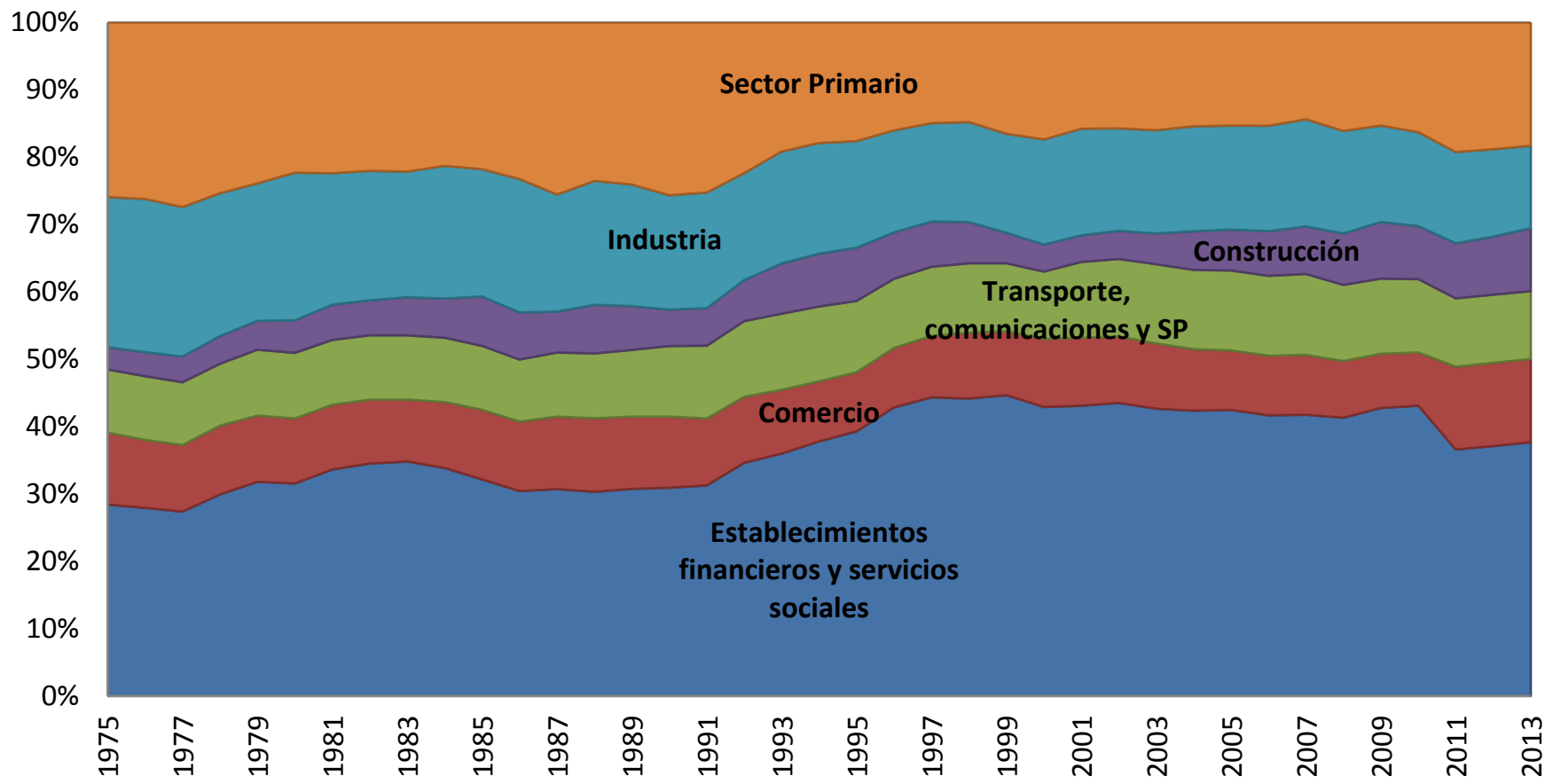

Figura 2. Evolución de la participación del PIB monetario por sectores económicos en Colombia: 1970-2013. Fuente: Banco de la República: www.banrep.gov.co.

La compilación de los datos se basó en una combinación de revisión de literatura (fuentes académicas y no académicas, incluidos los registros de activistas y de las mismas comunidades) y datos primarios recogidos por el autor y por las redes de apoyo de ONG's y de estudiantes al proyecto, a través de las visitas de campo y las entrevistas a lo largo de varios viajes de estudio realizados entre 2009 y 2014. Cada caso ha sido nombrado acorde a como es conocido en el discurso público en Colombia y los resúmenes de la mayoría de los casos se encuentran disponibles en la base de datos del proyecto EJOLT (www.ejolt.org).

Con la información obtenida en los formularios de EJOLT se construyó una base de datos que permitió clasificar, caracterizar y tipificar los conflictos. Con esta información se establecieron dos esquemas analíticos: i) A través del mapeo, que además de permitir la ubicación geográfica del conflicto, posibilitó el cruce de información con otras variables y mapas mejorando el análisis de los conflictos. ii) Para complementar el marco analítico se usó la estadística descriptiva para profundizar en el conocimiento de los conflictos.

Aunque se reconoce que la lista no es completa, está es más un inventario que una muestra, ya que proporciona una visión general, tanto de las actividades generadoras como del alcance geográfico de los conflictos ambientales de los últimos años en el país. Se han incluido casos bien documentados para asegurar una buena descripción, queriendo abarcar además buena parte del territorio colombiano y los principales productos, actividades y proyectos generadores de conflictos.

Los criterios para seleccionar cada conflicto corresponden al nivel de exigencia y visibilidad de los reclamos, críticas, movilizaciones y protestas que los afectados han expresado con respecto a una actividad, proyecto o producto específico (e.g. un punto de extracción de petróleo, un vertedero de residuos, etc.) que los afecta, con el propósito de conquistar sus derechos a una justicia ambiental.

\section{RESULTADOS: INVENTARIO DE LOS CONFLICTOS AMBIENTALES EN COLOMBIA}

En la Figura 3 (mapa), se presentan los 95 conflictos inventariados dentro de esta investigación. Como se observa, el inventario incluye conflictos de todo tipo: se ubican en 
buena parte de las regiones del país; son generados por diferentes tipos de actividades en distintos sectores de la economía (agropecuario o biomasa; infraestructura; energía fósil; fumigaciones; generación de energía; minería y residuos sólidos); incluyen las diferentes fases de los proyectos o actividades generadores de los impactos: propuesta, planeación y operación de los proyectos; afectan distintos tipos de comunidades (población urbana, campesinos, comunidades indígenas, comunidades afro descendientes); son generados por diferentes tipos de empresas (nacionales, extranjeras y mixtas); afectan distintos tipos de ecosistemas y de recursos naturales (tierra, agua, páramos, humedales, bosques, ríos, mares, manglares, etc.); la comunidad afectada desarrolla diferentes tipos de resistencias sociales (movilizaciones, paros, protestas, denuncias, etc.); recurren igualmente a diferentes tipos de instrumentos legales y jurídicos para defenderse y para buscar la justicia ambiental (actos legislativos; consultas populares; acuerdos municipales; tutelas; acciones de cumplimiento; derechos a consulta previa; etc.); entre otros.

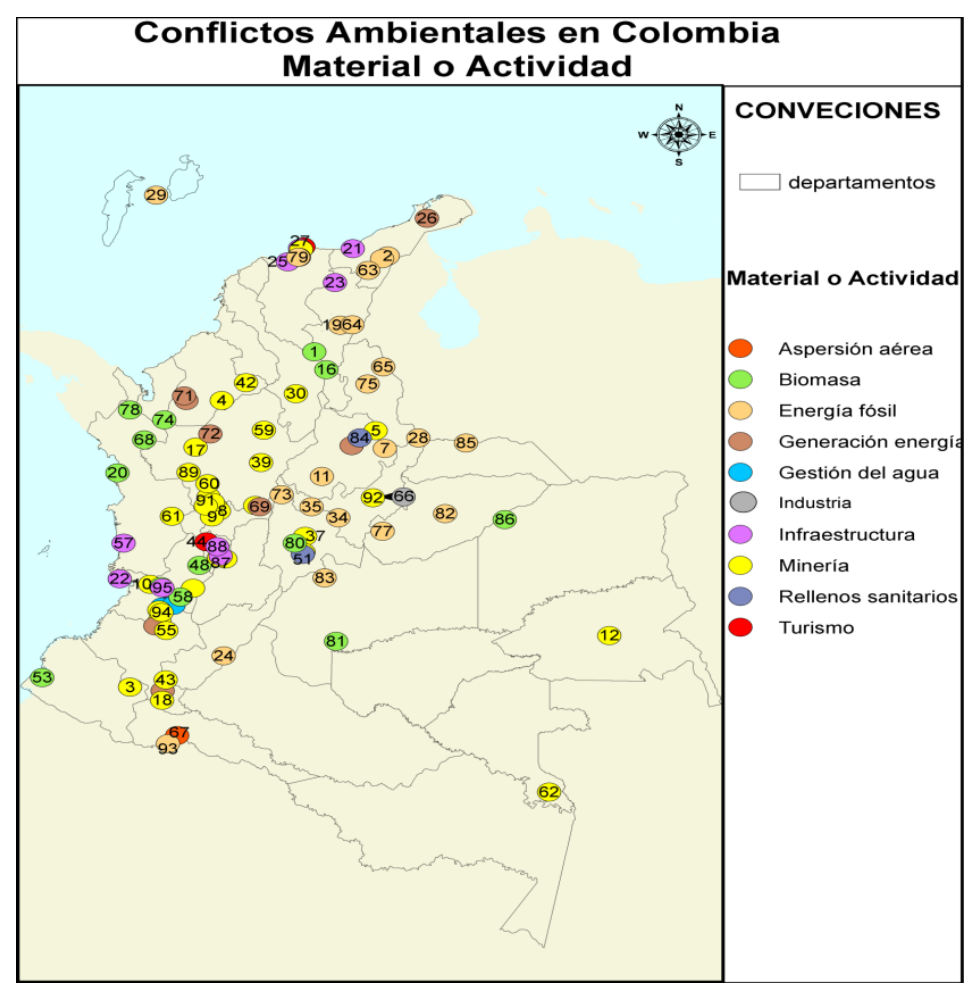

Figura 3. Mapa de los conflictos ambientales en Colombia Fuente: Base de datos Univalle-EJOLT (Colombia).

Ahora, para profundizar en el conocimiento de los conflictos, este capítulo desagrega el análisis en cuatro ejes temáticos:

i) Análisis espacial de los conflictos ambientales a partir de su ubicación georeferenciada. ii) Periodización de los conflictos ambientales.

iii) Impactos ambientales y sociales de los proyectos generadores de conflictos ecológico distributivos en Colombia.

iv) Resultados de los conflictos y mecanismos de resistencia de los afectados.

\section{La geografía de los conflictos ambientales en Colombia}

Para la ubicación espacial de los conflictos se realizaron varios gráficos descriptivos. Las Figuras 4 y 5 permiten identificar espacialmente los conflictos en la geografía colombiana.

Acá se puede observar que la mayor parte de los conflictos se presentan en la región Andina y en la Costa Caribe. En estas dos zonas se concentra el $90 \%$ de los habitantes del país, evidenciando que los conflictos se producen en donde la población se ve más afectada por el impacto ambiental o la pérdida de acceso al recurso natural. Así, de los 95 conflictos estudiados, 68 (72\%) se producen en esas dos regiones, 23 en el Caribe y 45 en la región Andina. En la región Andina los departamentos más afectados son Valle, Antioquia, y Santander. En la región Caribe son Magdalena, la Guajira y Córdoba.

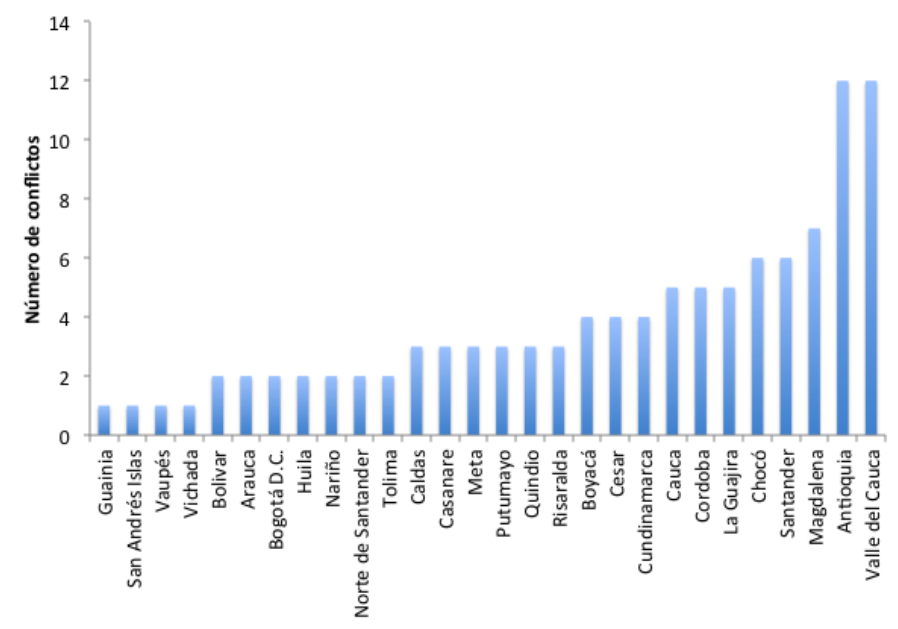

Figura 4. Principales conflictos socio-ambientales en Colombia y su ubicación por departamentos. Fuente: Proyecto Univalle-EJOLT (Colombia).

En términos de las actividades económicas generadoras de conflictos por regiones, la zona Andina (Figura 5) se caracteriza por conflictos de origen: minero, donde hay que resaltar los dos conflictos más simbólicos del país en zonas de alta montaña, ambos asociados con la extracción de oro: el del Páramo Santurbán (Santander) y el de La Colosa [Conflictos 5 y 13]; igualmente se destacan los relacionados con la generación de energía eléctrica: en esta región se desarrollan y operan los principales proyectos hidroeléctricos del país: El 
Quimbo (Huila); Hidrosogamoso (Santander); Represa Salvajina (Cauca); Hidromiel (Caldas); e Hidroituango (Antioquia) [Conflictos 14, 38, 40, 69 y 72]. Pero además, se presentan varios conflictos asociados a la extracción de energía fósil: en carbón se resaltan los conflictos en páramos como El Almorzadero (Santander), Guacheneque (Cundinamarca-Boyacá) y Rabanal (Cundinamarca) [Conflictos 7, 34 y 35]. En petróleo están los conflictos del páramo de Miraflores (Huila) y el conflicto de extracción de petróleo en territorio indígena Motilón-Bari [Conflictos 24 y 75].

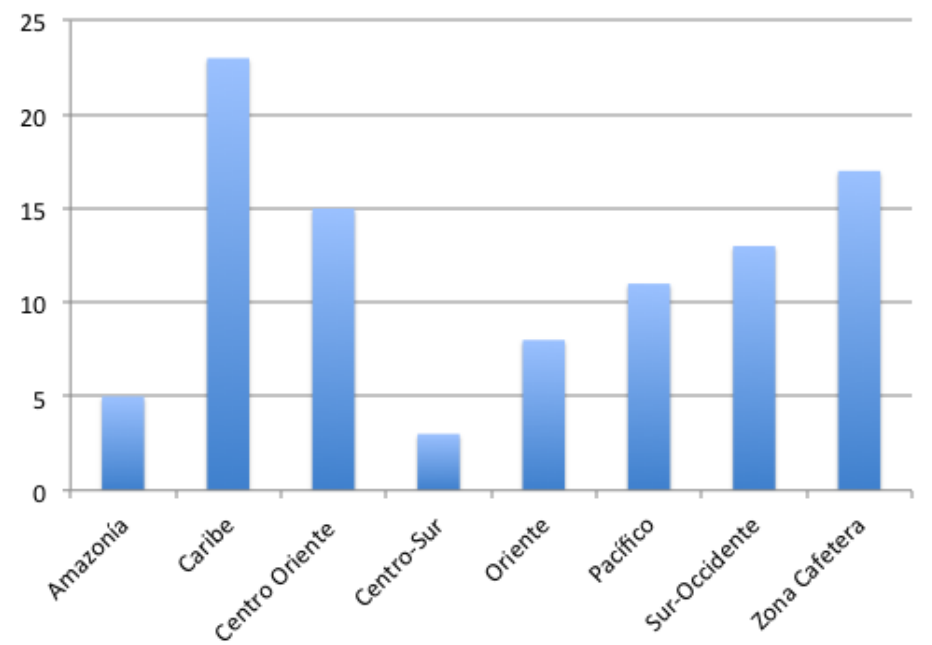

Figura 5. Conflictos socio-ambientales por regiones en Colombia. Fuente: Proyecto Univalle-EJOLT (Colombia).

En la región Caribe los principales conflictos son generados por las actividades de extracción de energía fósil, donde hay que destacar las minas de carbón de El Cerrejón (La Guajira), La Loma en la Jagua de Ibirico (Cesar) y las Barcazas Carbón Drummond. Son proyectos, generadores de importantes impactos ambientales y conflictos, no solo en su fase extractiva sino en el transporte y cargue hacia el exterior [conflictos 2, 19, 50 y 79]. Igualmente, sobresale el sector de la infraestructura, principalmente la construcción de puertos conflictos [21 y 27], y el conflicto más antiguo reportado, la carretera sobre la Ciénaga Grande de Santa Marta [25]. También predominan los conflictos por actividad minera [conflictos 4, 30, 36, 42 y 63], donde ocupa lugar preponderante el de Cerromatoso (Córdoba) [4], una de las minas de ferroníquel más grandes de América Latina. En la región Pacífica se identificaron 11 conflictos, cuatro asociados a minería [10, 17, 61 y 94], cuatro a biomasa [20, 53, 68 y 78], donde se destaca la palma, y tres de infraestructura, dos en puertos, el de Tribugá en el Chocó [57] y el de Bahía Málaga en el Valle del Cauca [22] y uno de infraestructura de carretera, el de la carretera Mulaló-Loboguerrero [95]. En la Amazonía se presentan cuatro conflictos, dos vinculados a la minería [12 y 62], uno a la energía fósil [93] y el otro, un conflicto internacional entre Colombia y Ecuador, sobre fumigación de cultivos ilícitos [67].

En la Orinoquia se identificó seis conflictos de energía fósil, entre ellos el conflicto importancia internacional de las exploraciones petroleras en territorio indígena U’wa [28] y uno de Biomasa, en la Macarena [81].

\section{Periodización de los conflictos ambientales}

En general hay consenso entre los académicos, de que el modelo de desarrollo económico en América Latina viró, a partir de finales de los sesenta, desde la industrialización inducida por la sustitución de importaciones con miras a consolidar el mercado interno (desarrollo "hacia adentro"), hacia la promoción de exportaciones y la búsqueda de demandas en el sector externo (desarrollo "hacia fuera") (Ocampo 1993, GRECO 2002, Kalmanovitz \& López 2006, Ortíz 2009, Bielschowsky et al. 2011).

Este marco analítico permite mirar comparativamente la dinámica de los conflictos ambientales alrededor de las dinámicas de especialización productiva de la economía colombiana y de las políticas que los diferentes gobiernos han promovido para impulsar la actividad económica en el país. Con este propósito se divide el análisis temporal de los conflictos en 4 periodos: antes de 1990, para abordar el periodo previo a la apertura económica; entre 1990 y 2001, para identificar la dinámica de los conflictos posteriores a la apertura económica y previos al gobierno de Uribe Vélez que fue el que más flexibilizó la entrada de capitales al sector extractivo y liberalizó la titularización de territorios para estas actividades. Precisamente, la siguiente fase de análisis hace referencia al periodo 2002-2010 que corresponde a los dos periodos presidenciales del expresidente Uribe. Finalmente, para conocer la dinámica de los conflictos del gobierno Santos, se estudia el periodo que corresponde a después de 2010.

La Figura 6 muestra la dinámica de desarrollo de los conflictos socio-ambientales estudiados, encontrando que es a partir del año 2000, y en particular desde 2002, que se dinamiza la generación de este tipo de conflictos en Colombia. Así se observa que hasta antes de 1994 se habían desarrollado 20 conflictos, número que se fue acrecentando durante los siguientes periodos, hasta alcanzar los 21 conflictos que se generaron durante el primer gobierno de Uribe Vélez (2002-2006) y los 34 de su segundo periodo presidencial (2006-2010). Durante el presente gobierno, ya se han generado 9 conflictos ambientales.

Esta dinámica evidencia los resultados del modelo reprimarizador y neo-extractivista de la economía 
colombiana, que se desarrolla con fuerza a partir del primer gobierno de Uribe, adquiere su colofón en el segundo, y continúa bajo el gobierno actual. Mientras en los ocho años del gobierno uribista esta política se sustentó en la denominada "Confianza Inversionista", en el gobierno actual, con un espíritu similar, se soporta en la llamada "Locomotora minero-energética"; ambas tienen como una de sus estrategia facilitar la inversión extranjera para aprovechar las ventajas comparativas del país en términos de la abundancia de recursos naturales.

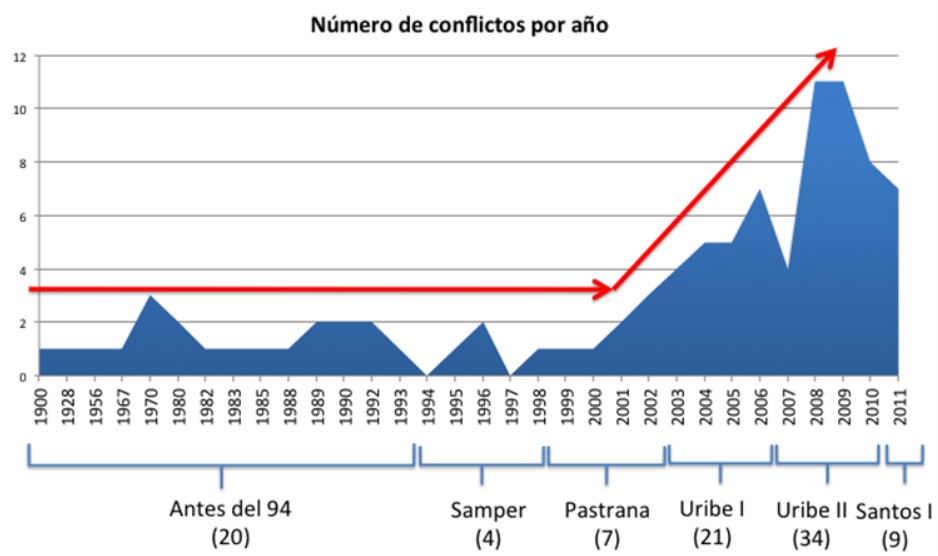

Figura 6. Periodización del inicio de los Conflictos ambientales en Colombia. Fuente: Proyecto Univalle-EJOLT (Colombia).

Precisamente la Figura 7, permite relacionar la dinámica de la inversión extranjera en Colombia con la generación de conflictos ambientales. Se evidencia en la gráfica una estrecha relación entre ambos aspectos, de tal manera que a medida que ha ingresado más capital extranjero al país para invertir en la actividad económica, más conflictos socio-ambientales se han generado. Es claro señalar también que estos conflictos tienen que ver con el destino de la inversión extranjera directa (IED), que como ya se observó en la Figura 5, esta se ha dirigido en forma creciente hacia el sector primario hasta alcanzar un 54\% del total para 2012 (US\$ 8300 ). Se sabe que el sector primario por definición es un usuario intensivo de recursos naturales, un acaparador y concentrador importante de naturaleza y un gran generador de impactos ambientales, obteniendo como corolario una dinamización de los conflictos ecológicos distributivos en el país.

Impactos ambientales y sociales de los proyectos generadores de conflictos ambientales en Colombia

Otro aspecto importante a estudiar en esta caracterización de los conflictos ecológico distributivos, son los impactos ambientales y sociales que generan los proyectos $\mathrm{o}$ actividades creadoras de conflictos. En el primer aspecto se quiere identificar cuáles recursos naturales y ecosistemas son los más afectados por estos proyectos; en el segundo caso, observar los principales grupos sociales impactados.

- Impactos ambientales de proyectos o actividades generadoras de conflictos

Hay diferentes maneras de ver los impactos ambientales creados por los proyectos o actividades generadoras de los conflictos. En este caso se abordarán por dos vías: el principal recurso natural afectado y el principal ecosistema afectado.

Cuando se analizan los conflictos acorde al principal recurso natural y ecosistema afectado se encuentran datos de interés. Por un lado, el principal recurso afectado es el agua con un $32 \%$ de los casos; seguido por la biodiversidad con $21 \%$; el paisaje y el suelo tienen igualmente una participación del 21 y $20 \%$ respectivamente. Finalmente el aire es afectado en un $6 \%$ de las veces por los proyectos generadores de conflictos (Figura 8). Estas cifras coinciden con el principal ecosistema afectado que son los ríos (32\%), después los bosques con un $25 \%$; los páramos y los humedales con un 7 y $6 \%$, respectivamente. Otros ecosistemas tienen un $19 \%$.

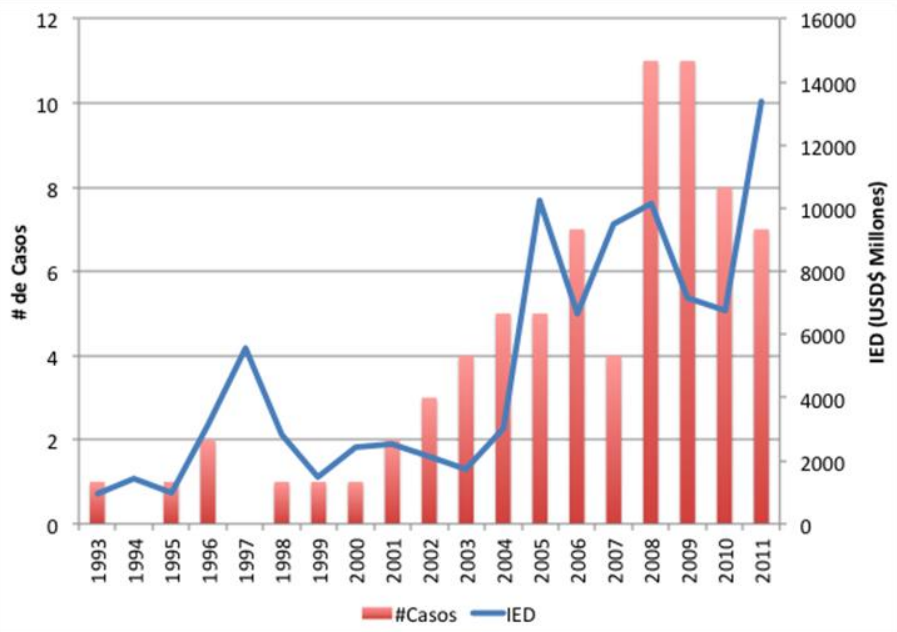

Figura 7. Inversión extranjera y conflictos ambientales en Colombia. Fuente: Proyecto Univalle-EJOLT (Colombia).

El otro gráfico evidencia igualmente lo que se afirmó antes: los ecosistemas más afectados están ubicados también en las zonas rurales, pero con una conexión importante, en algunos casos, con las zonas urbanas. Estos corresponden a los páramos que proveen agua a varios sistemas de acueducto de las zonas urbanas (Santurbán, Guacheque y Rabanal [5, 34 y 35]).

- Grupos humanos afectados por los proyectos o actividades generadoras de conflictos

Los principales grupos humanos afectados por los conflictos 
corresponden a los sectores más pobres de la población. En la Figura 9 se observa que el principal colectivo social afectado es el campesinado, con un porcentaje de afectación de $23 \%$. El segundo grupo humano impactado son los habitantes de las ciudades con un indicador de afectación de 15\%; sin embargo, la cantidad de personas afectadas para este grupo poblacional es mayor por su densidad poblacional. Los indígenas alcanzaron un índice de afectación de 14\%. La comunidad afrodescendiente por su parte, alcanzó un nivel de afectación relativamente bajo de $6 \%$, superado por los pescadores que alcanzaron el $10 \%$ y los pequeños mineros con $7 \%$. Finalmente están los turistas con el $5 \%$ y las empresas que son afectadas por otras empresas tienen un índice de afectación del 5\% de los casos (Figura 9).

Ahora, cuando se examinan el número de conflictos donde hay afectación de comunidades indígenas y afrodescendientes el porcentaje se incrementa. Así, de los 95 casos de conflictos en el territorio, la mayoría - el $62 \%$ - de esos conflictos ocurren en zonas con presencia de pueblos indígenas y de comunidades afrocolombianas. $\mathrm{O}$ sea 58 conflictos inciden en zonas de pueblos étnicos. El 44\% afecta a pueblos indígenas y el 18\% a afrodescendientes. Los ejemplos sobran: el Cerrejón, el territorio U’wa y la exploración de la Oxy, los Embera-Katio y la represa de Urra, los puertos Brisa, Tribugá y Bahía Malaga, los proyectos turísticos en el Parque Nacional Tayrona, la actividad minera en el Macizo Colombiano, el coltán en Guainía, etc. Todos ocasionan violaciones del derecho a la vida, a la salud o a la autonomía (Figura 10).

Esto es lo que se ha denominado en la literatura como "racismo ambiental", o sea que las actividades extractivas o generadoras de impactos ambientales se ubican en los sitios donde viven las comunidades más pobres y marginadas o determinados tipo de etnias. Como lo señala el artículo del diario "El Espectador", "estas preocupantes cifras, obligan a centrar nuestra atención en tres puntos esenciales: la expansión de la frontera de explotación, la importancia de proteger el derecho a la consulta previa y la necesidad de generar alianzas entre los afectados". ${ }^{1}$ Pues lo grave, es que debido a esa infinidad de proyectos generadores de conflictos e impactos ambientales, varios de esos grupos étnicos fueron expulsados de sus territorios.

\section{Resultados de los conflictos y mecanismos de resistencia de los afectados}

La búsqueda de nuevas formas de acumulación de capital por parte de las fuerzas empresariales, amplia las fronteras

\footnotetext{
${ }^{1}$ EL ESPECTADOR. “Afros e indígenas los más afectados. Conflictos ambientales se dispararon con Uribe y Santos. Abril 7 de 2014.
}

económicas hacia nuevos territorios habitados por comunidades de diferente tipo: indígenas, campesinos, afrodescendientese incluso habitantes de las ciudades. En este proceso se generan diferentes tipos de impactos sobre estas comunidades que ven afectados sus medios de vida, sus valores culturales y sus formas de relacionarse consigo mismo y con la naturaleza. Por esta razón, las comunidades buscan diferentes mecanismos y estrategias de defensa y resistencia de sus derechos y su acceso a los servicios que la naturaleza les ofrece. Dentro de estos mecanismos de resistencia se encuentran las manifestaciones, los paros, los bloqueos, las gestiones institucionales y técnicas, el marco legal y jurídico y en algunas ocasiones las acciones de hecho.

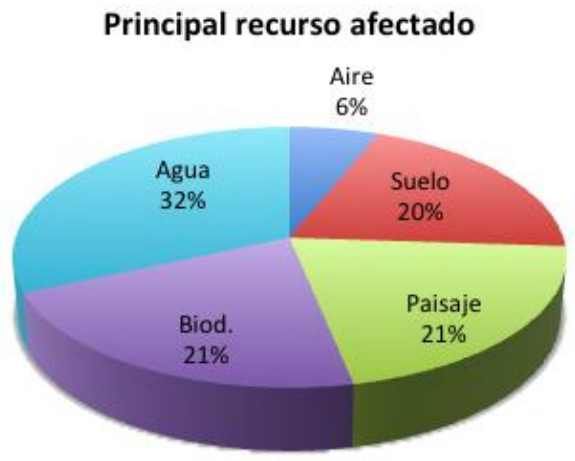

Principal Ecosistema afectado

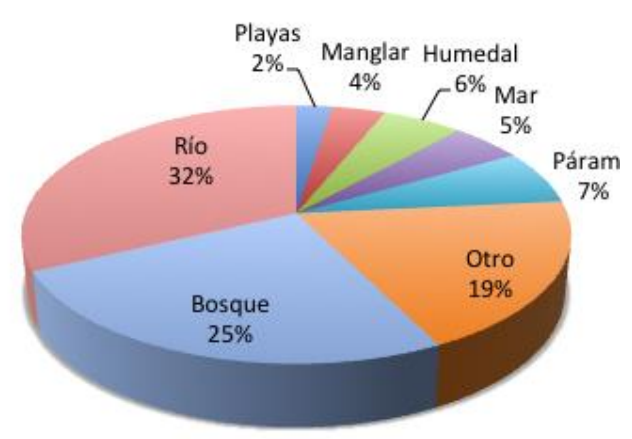

Figura 8. Clasificación de los conflictos acordes a los recursos naturales y ecosistemas afectados. Fuente: Proyecto UnivalleEJOLT (Colombia).

Pero igualmente, las empresas y fuerzas productivas también despliegan estrategias y mecanismos para preservar, fomentar y mantener estas nuevas fuentes de acumulación de capital y extraer los recursos naturales requeridos para el desarrollo metabólico del proceso capitalista. Estas estrategias combinan el marco legal, los estudios técnicos, las relaciones de poder y de política, las redes de influencia, la corrupción, las "puertas giratorias" 2 y en bastantes ocasiones el uso de la

\footnotetext{
${ }^{2}$ Corresponde al traslado de funcionarios del Estado de alto nivel hacia las direcciones o puestos de confianza de las empresas extractivas.
} 
violencia, ya sea legal, en manos del poder del estado a través de la fuerza pública, o ya sea ilegal en manos de grupos al margen de la ley o de las denominadas fuerzas oscuras. Similarmente, al lado de los empresarios extractivistas está el lenguaje del "desarrollo", del interés nacional, del beneficio general, en contra de los intereses locales y de grupos específicos de población que defienden su cultura, sus valores y sus medios de subsistencia.

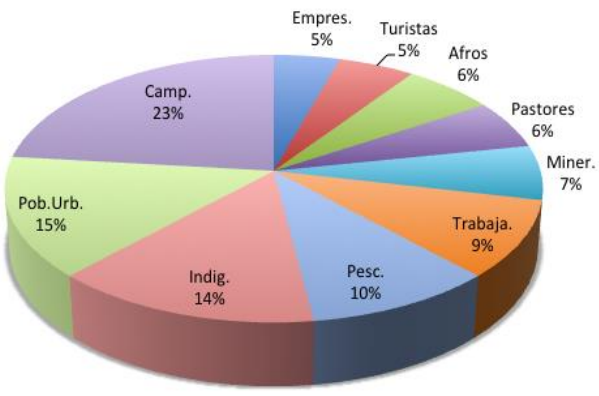

Figura 9. Principales grupos humanos afectados por los conflictos. Fuente: Proyecto Univalle-EJOLT (Colombia).

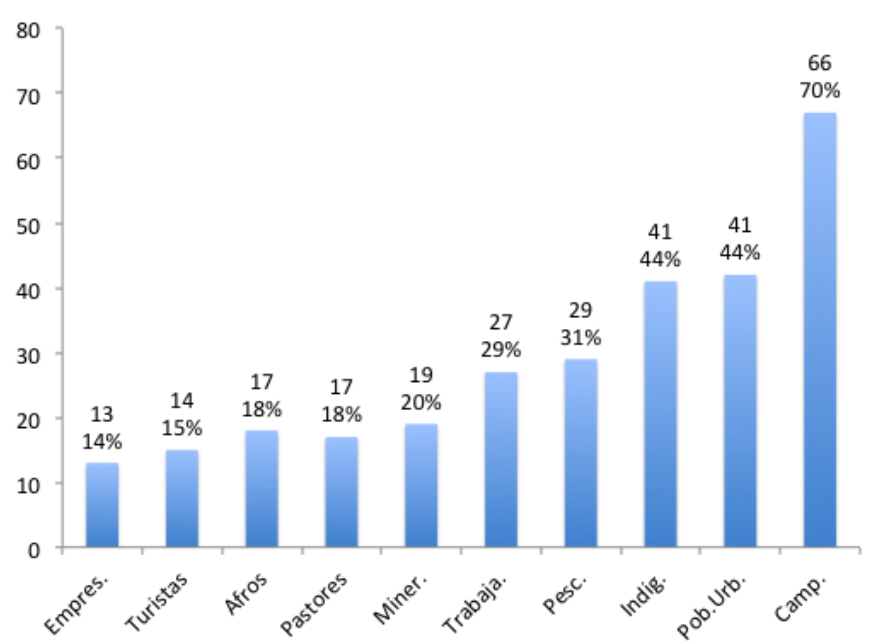

Figura 10. Conflictos ambientales por población afectada (\# de conflictos donde hay presencia de grupos poblacionales afectados/No son excluyentes). Fuente: Proyecto UnivalleEJOLT (Colombia).

En esta lucha se evidencian las asimetrías del poder político y económico: los "pudientes" o sea los que pueden, al lado del "desarrollo", de la "modernidad", del Estado, de las leyes hechas a su imagen y semejanza y en últimas del "estatus quo"; los otros, los marginados, los pobres, los vencidos por la historia, acompañados de las Organizaciones de Justicia Ambiental (OJA's) y en ocasiones arañando el marco legal y jurídico y el apoyo internacional, y usando su capacidad organizativa y comunitaria. Es una lucha desigual, pero es una lucha que expresa las resistencias de las comunidades tradicionales frente al avance del metabolismo social y del desarrollo.

Como consecuencia de estas disputas que tienen de por medio a la naturaleza, se produce una serie de resultados frente al proyecto generador del conflicto, el cual puede continuar, puede paralizarse, pueden producirse acuerdos entre los afectados y las empresas generadoras del conflicto, puede haber compensaciones a los afectados, pueden producirse hechos de violencia que nieguen al otro, lo desaparezcan, lo intimiden. También los afectados pueden recurrir al marco legal y jurídico existente para tratar de frenar, parar, o negociar el proyecto.

Precisamente en este punto, se caracterizan los resultados encontrados a este respecto a los 95 conflictos estudiados. Así, en este acápite se analizan dos cuestiones centrales: i) Las acciones resultantes o consecuencias finales de los conflictos; $y$, ii) las herramientas jurídicas utilizadas por los afectados para defender sus derechos.

- Acciones resultantes o consecuencias finales de los conflictos

Con respecto a las acciones, consecuencias o estrategias como se han resuelto o querido resolver los conflictos se tienen resultados paradójicos. Se observa una gran bipolaridad en las soluciones o resultados finales de los conflictos: por un lado, un gran número de acciones dirigidas a la negociación o el uso de instrumentos jurídicos; y por otro, una gran tendencia a resolver los conflictos o demandas de las comunidades a través de las acciones violentas. En la Figura 16 se observa este panorama: 165 casos en que se han usado herramientas pacíficas para intentar resolver el conflicto incluyendo: demandas en los tribunales (27), criterios técnicos (27), aplicación de la norma (38), compensaciones (23) y negociación (50).

Pero al mismo tiempo se observan 151 casos donde las soluciones se establecen por mecanismos violentos. En estas alternativas se destacan las amenazas a activistas (52), la represión (33), el desplazamiento (31), las muertes en 22 de los casos, lo que representa una tasa de $23 \%$ de todos los conflictos analizados, cifra bastante elevada; y desapariciones en 13 de los casos, cifra también considerable (Figura 11).

Sin embargo, otro dato rescatable es que 19 proyectos se han logrado detener por diferentes aspectos, alcanzando así una tasa de éxito del $20 \%$, ya sea parcialmente o en forma definitiva. Esto es lo que se ha denominado "triunfos de la 
justicia ambiental". Algunas de estas detenciones se han logrado por el movimiento social generado alrededor del proyecto y otras por aspectos relacionados con el tema legal, o el vencimiento de términos del proyecto.

- Instrumentos legales usados por los afectados

Ahora, con relación a los instrumentos legales utilizados para intentar resolver los conflictos, se encuentra la siguiente información condensada en la Figura 12. Dentro del marco jurídico utilizado por los afectados, se destaca la normativa ambiental como el recurso más utilizado con un total de 44 casos. Esto representa casi la cuarta parte de los instrumentos usados en los diferentes sectores.

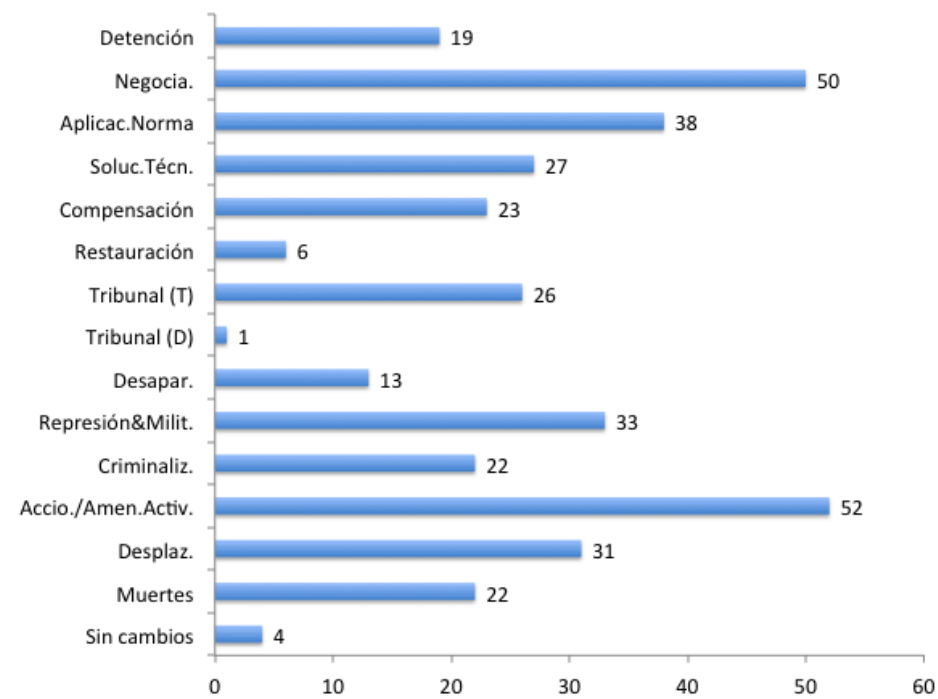

Figura 11. Acciones resultantes de los conflictos (\# de eventos presentados/No son excluyentes). Fuente: Proyecto UnivalleEJOLT (Colombia).

Por su parte, las acciones dirigidas a defender el derecho a la consulta previa existente en la legislación colombiana son también un recurso legal importante; en este caso se hace referencia a la Ley 70 y al Convenio 169 de la OIT. Estos instrumentos obligan a que deban ser consultadas las comunidades afrodescendientes e indígenas antes del desarrollo de un proyecto. Las herramientas señaladas representan casi la tercera parte del total de instrumentos legales usados por las comunidades para defender sus intereses.

De otro lado, se destaca igualmente la Acción Popular que se presenta en 6 ocasiones donde se reconoce el caso del acueducto de Pance. También la Tutela ha sido usada en 7 ocasiones, una de ellas en el conflicto minero en Titiribí, Antioquia [60]. Estos son dos instrumentos de la legislación colombiana, que sirven para defender los derechos colectivos e individuales de los afectados (Figura 12).
A nivel de Consultas Populares o Plebiscitos, se han presentado cinco que buscaban detener los desarrollos extractivos en sus regiones. Se trató de proyectos mineros: el del páramo de Santurbán [5], que resultó relativamente exitoso, la consulta en el municipio de Piedras que votó negativamente el uso de su territorio para actividades mineras en el proyecto La Colosa [13], la consulta en Tauramena [77] y en los municipios de Urrao [89] y El Jardín [91]. Estas iniciativas han generado un conflicto jurídico entre la autonomía de los municipios y el interés de la Nación frente al uso del territorio. Ahora, en términos de Acuerdos Municipales anti-extractivistas, son diecisiete los establecidos para detener proyectos: Cinco en minería [8, 13, 89, 90 y 91], seis acuerdos en actividades de extracción de biomasa [56, 58, $76,78,81$ y 86], cinco de energía fósil [7, 75, 82, 83 y 85] y uno de relleno sanitario [84].

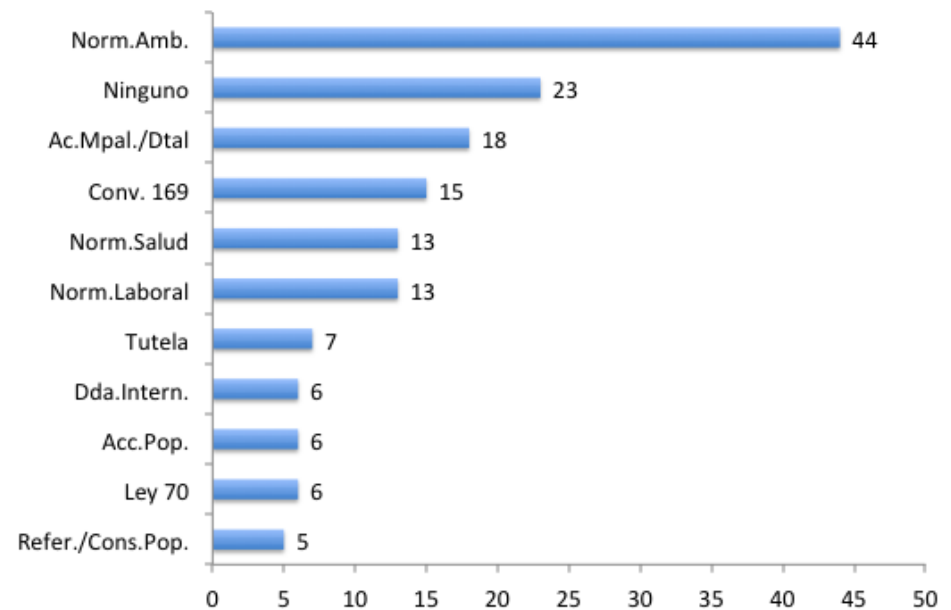

Figura 12. Instrumentos legales usados por los afectados Nota: \# de veces que se reporta este instrumento. Fuente: Proyecto Univalle-EJOLT (Colombia).

Ahora, si se detalla esta información por sectores económicos se encuentra que: el sector que más ha usado instrumentos legales es el de la minería (29\%), seguido de la energía fósil con $28 \%$, la biomasa $16 \%$, la infraestructura con un $13 \%$ y la generación de energía y rellenos sanitarios con $11 \%$ cada uno. El resto tienen participaciones minoritarias. En la minería se ha usado esencialmente normativa ambiental (10), acuerdos municipales (5), normativa laboral (4) y referendos y consulta popular (4). En energía fósil la normativa ambiental (15); en biomasa acuerdos municipales (6); y en residuos sólidos la normativa ambiental y de salud (6) (Tabla 1).

\section{CONCLUSIONES}

Los resultados obtenidos por esta investigación permitieron concluir que: 
- Colombia ha acentuado su proceso de especialización productiva hacia el sector primario. Existe suficiente evidencia estadística de esta situación: en Colombia, las exportaciones de origen primario incluyendo las manufacturas basadas en recursos naturales pasaron de presentar el 78\% del total de ventas externas al 84\% entre 1990 y 2013. Pero además, se ha producido un cambio estructural en el sector primario: caen las exportaciones agropecuarias y se incrementan en forma ostensible las minero-energéticas. Las primeras pasan del 60 al 3\% entre 1975 y 2012 y las segundas aumentan en el mismo periodo del 9 al $64 \%$ del total de exportaciones. Esto igualmente se ve reflejado en el PIB: el sector agropecuario descendió en su participación del 23\% del PIB en 1975 al 6\% en 2012; mientras el sector mineroenergético incrementó su participación en el ingreso total nacional del $2 \%$ al $11 \%$ en el mismo periodo (DANE. 2013). Estos datos evidencian un fuerte proceso de reprimarización de la economía colombiana hacia la minería y los hidrocarburos.

- Buena parte de las inversiones nacionales y extranjeras se ejecutan ampliando las fronteras económicas hacia nuevos territorios. Esto se refleja en la ampliación de la frontera agrícola; la frontera forestal; la frontera minera; la frontera petróleo-energética; la frontera palmera; la frontera cañera.

- La ampliación de las fronteras económicas hacia territorios con usos tradicionales o ricos en ecosistemas estratégicos, incrementa las presiones sobre el ambiente e intensifica los conflictos ambientales. La política minera. energética y agrícola impulsada por los últimos gobiernos en Colombia ha potenciado el modelo extractivo y ello ha implicado un cambio drástico en el uso del suelo que choca con los planes de vida de comunidades indígenas, campesinos, pescadores y afrodescendientes que ancestralmente han habitado y conservado el equilibrio en sus territorios, poniendo en riesgo la sobrevivencia de diversas especies, la disponibilidad de agua para consumo humano, las formas de vida $y$ sobrevivencia de comunidades urbanas y rurales. Muchas de las nuevas actividades se ubican en estos territorios generando o intensificando los conflictos ambientales.

- Buena parte de los conflictos se ubican en las zonas más pobladas (Andina y Caribe) y muchos en zonas de conservación. Ello haya explicación en que buena parte de las actividades extractivas se desarrollan en zonas de alta densidad demográfica como la zona Andina, pero igualmente se expanden las fronteras hacia nuevos territorios donde existen zonas de conservación como parques nacionales, territorios de comunidades indígenas y afros.

- Las nuevas fronteras de expansión de la actividad económica generadora de conflictos se ubican más en zonas cercanas a centros poblados y a la infraestructura para la extracción. Por tal razón y por la abundancia de ciertos recursos mineros como el oro, las nuevas zonas de expansión se ubican en la zona Andina y el Caribe. En ambos casos, las fronteras de colonización son los páramos, el Magdalena Medio, la zona de Urabá y otras más. En términos de la cantidad de conflictos, la futura frontera parecería más la zona Pacífica y el Chocó biogeográfico que la Orinoquía y la Amazonía. Aunque con las nuevas estrategias de desarrollo hacia el sector agropecuario y la abundancia de petróleo está próximo a entrar la Orinoquía colombiana como nueva frontera económica. Las noticias recientes confirman esta hipótesis.

- Hay una clara relación entre la cantidad e intensidad de los conflictos ambientales y el modelo de desarrollo extractivo de los últimos gobiernos. La apertura económica y la desregulación de la mayor parte de las actividades productivas y extractivas por parte del estado en la década del noventa, sentó las bases del modelo extractivista acrecentado en el presente siglo. Se resalta en este caso los dos gobiernos de Uribe Vélez que concentran el 58\% de todos los conflictos analizados, los cuales se ubican en casi todos los sectores: minería, puertos, hidroeléctricas, energía fósil y biomasa. Esta situación es alimentada por la dinámica de la Inversión Extranjera Directa y la flexibilización de las normativas para la asignación de territorios para el desarrollo de actividades extractivas y el desmonte institucional ambiental y minero-energético.

- Los dos gobiernos de Uribe Vélez concentran la mayor parte del área y la población afectada por los proyectos de desarrollo extractivo. Así, este periodo acumula el $42 \%$ del total del territorio afectado y el $53 \%$ de las personas impactadas que equivalen a 5.8 millones.

- El sector extractivo explica buena parte de los conflictos socio-ambientales del país donde se destaca la minería, la biomasa y la energía fósil. La actividad minera concentra el $34 \%$ de los conflictos, destacándose dentro de ella el oro con el $29 \%$ del total. La energía fósil acumula el $20 \%$ de los conflictos, encabezada por el carbón que representa el 13\% del total de conflictos. Por su parte, la biomasa explica el 8\% de los conflictos, donde se destaca la palma y la caña.

- Los principales recursos y ecosistemas afectados por los proyectos generadores de conflictos son el agua y el suelo. y los ríos y bosques. Así, entre los tres primeros recursos se concentran el $74 \%$ de los impactos y entre los dos primeros ecosistemas el $57 \%$ de los mismos. El sector económico que más afecta ambos tipos de recursos y de ecosistemas es la minería. 
Tabla 1. Marco legal utilizado por las comunidades afectadas en la búsqueda de la Justicia Ambiental en Colombia. Fuente: Base de datos EJOLT (Colombia).

\begin{tabular}{|c|c|c|c|c|c|c|c|c|c|c|c|c|c|}
\hline Período & $\begin{array}{c}\text { Refer./ } \\
\text { Consulta } \\
\text { Popular }\end{array}$ & Ley 70 & $\begin{array}{c}\text { Conv. } \\
169\end{array}$ & $\begin{array}{l}\text { Acc. } \\
\text { Pop. }\end{array}$ & $\begin{array}{c}\text { Ac.Mpal./ } \\
\text { Dtal }\end{array}$ & Tutela & $\begin{array}{l}\text { Norm. } \\
\text { Amb }\end{array}$ & $\begin{array}{l}\text { Norm. } \\
\text { Laboral }\end{array}$ & $\begin{array}{l}\text { Norm. } \\
\text { Salud }\end{array}$ & $\begin{array}{l}\text { Dda. } \\
\text { Intern. }\end{array}$ & Ninguno & Total & $\%$ \\
\hline Aspersión aérea & & & & & & & & & 1 & 1 & & 2 & $1.28 \%$ \\
\hline Biomasa & & 2 & & 1 & 6 & 3 & 4 & 3 & 2 & 2 & 2 & 25 & $16.03 \%$ \\
\hline Energía fósil & 1 & 0 & 7 & 2 & 5 & 1 & 15 & 4 & 5 & 2 & 1 & 43 & $27.56 \%$ \\
\hline $\begin{array}{l}\text { Generación } \\
\text { energía }\end{array}$ & & 1 & 3 & & & & 7 & & & & & 11 & $7.05 \%$ \\
\hline Gestión del agua & & & & 1 & & & & & 1 & & & 2 & $1.28 \%$ \\
\hline Industria & & & & & & & & 1 & 1 & & & 2 & $1.28 \%$ \\
\hline Infraestructura & & 2 & 2 & 1 & 0 & 1 & 4 & & & & 3 & 13 & $8.33 \%$ \\
\hline Minería & 4 & 1 & 3 & & 5 & 1 & 10 & 4 & & 1 & 16 & 45 & $28.85 \%$ \\
\hline $\begin{array}{l}\text { Rellenos } \\
\text { sanitarios }\end{array}$ & & & & 2 & 1 & 1 & 3 & 1 & 3 & & & 11 & $7.05 \%$ \\
\hline Turismo & & & & & & & 1 & & & & 1 & 2 & $1.28 \%$ \\
\hline Total & 5 & 6 & 15 & 7 & 17 & 7 & 44 & 13 & 13 & 6 & 23 & 156 & $\begin{array}{r}100.00 \\
\%\end{array}$ \\
\hline$\%$ & $3.21 \%$ & $3.85 \%$ & $9.62 \%$ & $4.49 \%$ & $10.90 \%$ & $4.49 \%$ & $28.21 \%$ & $8.33 \%$ & $8.33 \%$ & $3.85 \%$ & $14.74 \%$ & $100.00 \%$ & \\
\hline
\end{tabular}


- En términos de impactos sociales, estos recaen sobre los grupos humanos más pobres y marginados. Campesinos (23\%). indígenas $(14 \%)$. pequeños pescadores $(10 \%)$. pequeños mineros $(7 \%)$ y comunidades afrodescendientes (6\%) son los más afectados. El sector que más afecta a estos grupos humanos es la minería.

- Los resultados e intentos de solución de los conflictos muestran la bipolaridad de la sociedad colombiana. Por un lado. un gran número de acciones asociadas a medios pacíficos como la negociación, el estudio técnico o el uso de instrumentos jurídicos; por otro lado, un gran uso de los medios violentos para resolver los conflictos. Esto muestra la bipolaridad de la sociedad colombiana que se enmarca en los extremos entre negociación, juridicidad y violencia.

- Dentro de los métodos pacíficos e institucionales, la negociación es uno de los mecanismos más utilizados para intentar resolver los conflictos ambientales. Dentro de estos métodos se identificaron 165 casos que incluyen las demandas en los tribunales (27), los criterios técnicos (27), la aplicación de la norma (38), las compensaciones (23) y la negociación (50).

- El uso de la violencia legal e ilegal que afecta derechos humanos fundamentales, sigue siendo un instrumento importante para la resolución de los conflictos ambientales en Colombia. Se presentan 151 eventos de violencia destacándose las amenazas a activistas (52), la represión (33). el desplazamiento (31), las muertes en 22 de los casos, significando ello una tasa de $23 \%$; las desapariciones están presentes en 13 de los conflictos, cifra también elevada. Los medios violentos son principalmente usados en el caso de la minería, la energía fósil, la generación de energía y la extracción de biomasa; los medios pacíficos se utilizan más en el sector de la infraestructura.

- Dentro de los instrumentos legales usados por los afectados, la normativa ambiental y los derechos a consulta previa son los más utilizados para tratar de detener los proyectos generadores de conflictos. El primero representa el $28 \%$, de los recursos legales utilizados entre la Ley 70 y el Convenio 169 de la OIT que obligan a consultar a las comunidades negras y las comunidades indígenas, en forma respectiva, cubren el $22 \%$ de las herramientas legales usadas por las comunidades afectadas.

- Las consultas populares, los acuerdos municipales y las herramientas que defienden la afectación de derechos colectivos o individuales son instrumentos legales que han venido popularizándose. Estos instrumentos han sido utilizados en 23 de los casos representando cerca del $24 \%$ del total de herramientas usadas. Se destaca los Acuerdos
Municipales con 17 casos y la acción popular con 7. Por su parte las Consultas Populares se han desarrollado en cinco oportunidades: Santurban [5]. Piedras (La Colosa) [13]. Taurema [77]. Minería en Urrua [89] y Minería en Jardín [91].

- Sin embargo, a pesar de la cantidad de eventos de movilización y resistencia y de los "triunfos" de la justicia ambiental, las voces de las comunidades son invisibilizadas sistemáticamente. Diversas personas han sido víctimas de represión. Persecución, Judicialización, amenazas, desaparición y asesinato. Por otra parte, en los lugares donde se han desarrollado los proyectos, miles de personas han visto desmejoradas las condiciones de vida en sus territorios por el aumento del costo de vida, las alteraciones de las cadenas productivas, la pérdida del trabajo, el rompimiento de tejidos sociales, las afectaciones irreversibles al ambiente y el aumento de conflictos sociales y ambientales.

\section{REFERENCIAS}

BANCO DE LA REPUBLICA. URL disponible en: www.banrep.gov.co

BIELSCHOWSKY. R, IZAM. M, \& MULDER. N. 2011. Dos estudios de la evolución del pensamiento de la CEPAL sobre la diversificación productiva y la inserción internacional (1949-2008). Series Comercio Internacional No112. CEPAL. Santiago de Chile.

CEPAL. 2013. (Fecha de acceso 24 de enero de 2013). URL disponible en: www.eclac.cl/comercio/SIGCI/

DANE - DEPARTAMENTO ADMINISTRATIVO NACIONAL DE ESTADÍSTICAS. 2013. Cuentas Nacionales Anuales e información Comercio Exterior. URL disponible en: www.dane.gov.co

DELBECQ, A. \& VAN DER VEN, A. 1971. A group process model for problem identification and program planning. Journal of Aplied Behavioural Science 7: 466-492.

EJOLT. 2012. Environmental Justice Organizations. Liabilities and Trade. (Fecha de acceso 2 de noviembre de 2012). URL disponible en: www.wjolt.org

FIERRO, J. 2012. La política minera en Colombia: la articulación y potenciación de conflictos alrededor de intereses privados. En: "Minería, territorio y conflicto en Colombia". 179-211. Universidad Nacional de Colombia. Facultad de Derecho. Ciencias Políticas y Sociales. Instituto Unidad de Investigaciones Jurídico-Sociales Gerardo Molina (UNIJUS). Bogotá. 
FUENTES, A. 2012. Legislación minera en Colombia y derechos sobre las tierras y los territorios. En: Minería, territorio y conflicto en Colombia: 215-232. Universidad Nacional de Colombia. Facultad de Derecho. Ciencias Políticas y Sociales. Instituto Unidad de Investigaciones Jurídico-Sociales Gerardo Molina (UNIJUS). Bogotá.

GRECO. 2002. El crecimiento económico colombiano en el siglo XX. Grupo de estudios de crecimiento económico del Banco de la República. Edición Fondo de Cultura Eco- nómica y Banco de la República. Bogotá.

GUDYNAS, E. 2010. Agropecuaria y nuevo extractivismo bajo los gobiernos progresistas de América del Sur. Territorios 5: 37-54.

HORNBORG, A. 1998. Towards an ecological theory of unequal exchange: articulating world system theory and ecological economics. Ecological Economics 25: 127-136.

KALMANOVITZ, S. \& E. LÓPEZ. 2006. La agricultura colombiana en el Siglo XX. Bogotá. Colombia: Fondo de Cultura Económica.

NADAL, A. 2011. Macroeconomic policies for sustainability. In: A. Nadal (Ed.). Macroeconomic policies. Livelihoods and Sustainability Policy Matters (18 ed): 12-49. International Union for Conservation of Nature and Natural Resources IUCN. Mexico.

OCAMPO, J. A. 1993. La internacionalización de la economía colombiana. En. Colombia ante la economía mundial. Compilado por M. Urrutia. TM Editores Bogotá, Colombia.

ORTÍZ, C. H. 2009. La desaceleración económica colombiana: se cosecha lo que se siembra. Revista de Economía Institucional. v.11. n. 21 (jul. / dic.).

OSBORN, A. 1953. Applied Imagination: Principles and Procedures of Creative Problem Solving. NewYork: Charles Scrbner's Sons.

SVAMPA, M. 2013. Consenso de los Commodities» y lenguajes de valoración en América Latina. Nueva Sociedad. 244: 30-46.

WALLERSTEIN, I. 1974. The Modern World System: Capitalist Agriculture and the Origins of the European World Economy in the Sixteenth Century. Academic Press, New York. $440 \mathrm{pp}$. 